Available online on 15.08 .2020 at http://jddtonline.info
ODDT * 5

Open $\odot$ Access

Research Article

\title{
Acute kidney injury with dark urine: the case of paroxysmal nocturnal hemoglobinuria
}

\author{
Kamel El-Reshaid *, Hasan Sabri **, Shaikha Al-Bader ** \\ * Department of Medicine, Faculty of Medicine, Kuwait University, Kuwait \\ ** Department of Medicine, kidney unit, Al-Amiri Hospital, Kuwait
}

\begin{abstract}
In this case report; we describe a patient with severe attack of paroxysmal nocturnal hemoglobinuria (PNH) following Ciprofloxacin therapy. He presented with recurrent abdominal pain, repeated vomiting and dark urine. Physical examination revealed severe pallor and jaundice. Laboratory investigations showed severe intravascular hemolysis with negative Coomb's test, progressive acute kidney injury (AKI), sterile blood cultures and negative serology for autoimmune diseases. Subsequently, he developed pancytopenia. Ham test was positive and flow cytometry, later on, confirmed PNH. He was supported with multiple transfusions of packed blood cells and hemodialysis. Ciprofloxacin was discontinued and his PNH was treated with Solumedrol $1 \mathrm{~g}$ daily for 3 days followed by Prednisone $60 \mathrm{mg} / \mathrm{day}$ for $2 \mathrm{months}$. Two weeks later; his hemolysis abated and his AKI improved. Up to 5 years later, he still has minor PNH clone yet without disease activity. In conclusion; our patient had acute drug-induced hemolytic crisis associated with minor PNH clone. With drug-vigilance; no further relapses were reported and his PNH clone remained stable for 5 years. The case expands the spectrum of PNH phenotypes and its triggering factors.
\end{abstract}

Keywords: AKI, anemia, autoimmune, flow cytometry, hemolysis, PNH.

Article Info: Received 03 June 2020; Review Completed 12 July 2020; Accepted 18 July 2020; Available online 15 August 2020

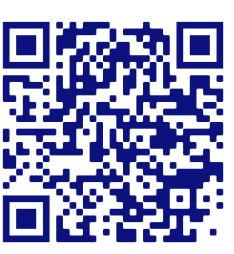

Cite this article as:

El-Reshaid K, Sabri H, Al-Bader S, Acute kidney injury with dark urine: the case of paroxysmal nocturnal hemoglobinuria, Journal of Drug Delivery and Therapeutics. 2020; 10(4-s):1-5 http://dx.doi.org/10.22270/jddt.v10i4-s.4196

*Address for Correspondence:

Kuwait

Dr. Kamel El-Reshaid, Professor, Dept. of Medicine, Faculty of Medicine, Kuwait University, P O Box 24923, 13110 Safat,

\section{INTRODUCTION}

Paroxysmal nocturnal hemoglobinuria (PNH) is a Coomb's negative intravascular hemolytic anemia characterized by attacks of hemolysis associated with dark urine due to severe hemoglobinuria ${ }^{1}$. It is an acquired nonmalignant clonal expansion of one or several hematopoietic stem cells that have acquired somatic mutation leading to loss of complement decay activating factor (DAF). The latter protects progeny of hematopoeitic cells from lysis by activated complements during innate immunological responses in infections, allergy and exposure to hapatens 2. It is a rare disorder with a prevalence of 0.5-1.5 per million people in the general population and with an average of 400 cases being diagnosed annually in USA ${ }^{3}$. Its multiple phenotypic forms and life-threatening complications mask its definitive diagnosis, alter its management and hence limit patients' survival ${ }^{4}$. In this case report; we describe a patient with this disorder and highlight the available means of diagnosis and management.

\section{THE CASE:}

A 45-year-old Egyptian man presented with abdominal pain vomiting and dark urine for 3 days. Past history was significant for fever and body aches 1 week ago for which Ciprofloxacin was used. On his initial physical examination; he was conscious and oriented X3. He had dyspnea on mild effort and tachycardia yet was afebrile and normotensive. He had severe pallor and jaundice. Initial and subsequent laboratory investigations are summarized in Table 1. Initially he had mildly decreased peripheral leucocytic and platelet counts yet severe anemia with low hemoglobin at 75 $\mathrm{g} / \mathrm{L}$, low serum haptoglobin and high reticulocytes\%. Serum urea and creatinine were elevated at $18 \mathrm{mmol} / \mathrm{L}$ and 503 umol/L, respectively. Serum glucose, electrolytes, albumin, ALT, alkaline phosphatase, GGT and amylase were normal. CPK was normal. Total and direct bilirubin, AST and LDH were high. Urine routine showed $4(+)$ blood yet on microscopy erythrocytes were $<5 / \mathrm{HPF}$. Stool testing was negative for ova, parasites and occult blood. Blood and urine culture were negative for pathogens. Blood film did not show significant abnormality except for fragmented RBCs 
"schistocytes" (Fig. 1). No malaria parasites were seen. Prothrombin and activated thromboplastin time were normal. Serum fibrinogen and procalcitonin levels were normal. Direct and indirect Coombs test were negative. G6PD level and hemoglobin electrophoresis were normal. Serum complements (C3 \& C4) and protein electrophoresis was normal. ANA, anti-ds DNA, ANCA, anti-GBM-antibodies, hepatitis $B$ surface antigen and anti-HCV antibodies were negative. Abdominal and pelvic ultrasound did not show abnormality and Doppler study did not show venous and arterial renovascular disease. Echocardiogram did not show abnormality. Ham test (sucrose lysis test) was positive and flow cytometry confirmed PNH later. Initially; ciprofloxacin was discontinued. His severe pancytopenia and progressive renal failure were treated with multiple blood transfusions and supportive hemodialysis. Since both did not improve by the $3^{\text {rd }}$ day; Solumedrol was started as $1 \mathrm{~g}$ IV daily for 3 days followed by Prednisone $60 \mathrm{mg}$ /day for 2 months. By 2 weeks; his clinical, hematological and biochemical abnormalities had improved and hence dialysis was discontinued. Two months after recovery; 24 hour urine testing for amino acids showed normal values. On follow up, and up to 5 years, his disease remained inactive with stable and minor PNH clone.

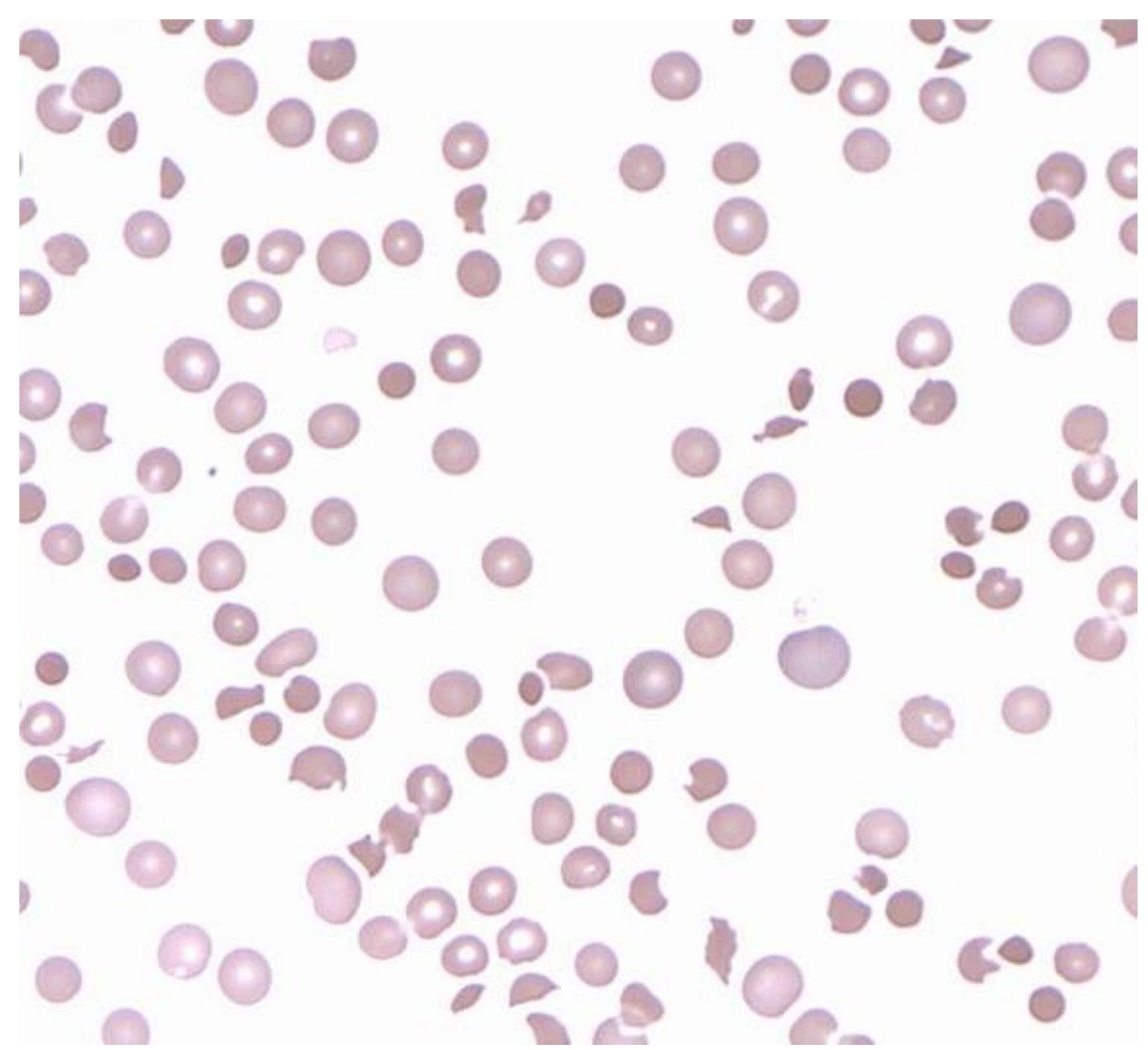

Figure 1: Peripheral blood smear showing schistocytes. 
Table 1: Initial and follow up laboratory investigations in the case of paroxysmal nocturnal hemoglobinuria (PNH)

Test/(Normal range)

\section{blood counts:}

Peripheral leucocytic count

$$
\left(4-10 \times 10^{9} / \mathrm{L}\right)
$$

Hemoglobin

$$
(130-160 \mathrm{~g} / \mathrm{L})
$$

Peripheral platelets count

$$
\left(150-450 \times 10^{9} / \mathrm{L}\right)
$$

Reticulocyte \%

$$
\text { (0.5-2.5\%) }
$$

Peripheral blood schistocytes

$$
\text { (Absent) }
$$

\section{Biochemistry:}

Haptoglobin

$$
\text { (0.5-2.2 g/L) }
$$

Serum creatinine

$$
\text { (64-104 umol/L) }
$$

Total bilirubin

$$
\text { (5-21 umol/L) }
$$

Direct bilirubin

$$
\text { (1-5 umol/L) }
$$

Aspartate aminotransferase (AST):

$$
\text { (3-35 IU/L) }
$$

Lactic dehydrogenase

$$
\text { (100-190 IU/L) }
$$

\section{Urine}

\section{routine:}

Blood

$$
\text { (absent) }
$$

\section{Flow cytometry:}

$\underline{\mathrm{RBC}}$

$$
\begin{aligned}
& \text { Type II (partial CD59 deficiency) } \\
& \text { Type III (complete CD59 } \\
& \text { deficiency) } \\
& \text { Total RBC PNH clone size }
\end{aligned}
$$

$0.80 \%$

$1.20 \%$

$4(+)$

$1(+)$

Negative

WBC-Neutrophils (FLAER/CD24

deficiency)

$1.30 \%$

WBC-Monocytes (FLAER/CD14 deficiency)

Diagnosis:
$1.80 \%$

Minor PNH

clone
$0.60 \%$

$1.80 \%$

$2.40 \%$

$2.60 \%$

$2.10 \%$

Minor PNH clone 


\section{DISCUSSION}

Our patient presented with progressive renal failure and severe intravascular hemolytic anemia. The increment in serum creatinine $>26$ umol/L within 48 hours indicated an AKI event 5. Moreover, the normal kidney ultrasound, Doppler study, lack of history of hypoperfusion, absence of urinary hematuria and proteinuria excluded pre-renal, postrenal, vascular, glomerular and autoimmune etiologies as well as an underlying chronic disease ${ }^{6}$. Previous Ciprofloxacin exposure had raised an issue of acute interstitial nephritis yet the clinical picture was far from being an isolated event. Though he had evident hemolysis and hemoglobinuria; testing 24 hour urine was done to exclude Alkaptonuria 2 months after clearance of hemoglobinuria 7. Work up for his severe anemia excluded; inherited membrane defect (G6PD), hemoglobinopathies (thalasemias and sickle cell disease), hereditary spherocytosis and elliptocytosis, falciparum malaria, and autoimmune ones ${ }^{8}$. Moreover, his anemia was (a) an acute event with high reticulocytes, (b) mediated by intravascular hemolysis with high direct bilirubin and LDH levels as well as intravascular with high hemoglobinemia and hemoglobinuria, (c) lacked autoimmune etiology with negative Coombs test, and (d) associated with normal bone marrow biopsy, and (e) associated with pancytopenia and blood "schistocytes" simulating microangiopathic picture. The latter was without malignant hypertension, scleroderma signs, disseminated intravascular coagulation (DIC) with prolonged coagulation tests and low fibrinogen, sepsis with fever and high procalcitonin levels 9. However, 2 microangiopathic disorders could not be ruled out with certainty viz. sporadic form of hemolytic uremic syndrome and thrombotic thrombocytopenic purpura. However, keeping a higher stage of diagnosis with severe intravascular hemolysis and AKI was safer to avoid side tracts with those 2 diagnoses. With hemoglobinuria; AKI is expected due to proteins liberated from hemolysed cells leading to vasoconstriction and nephrotoxic acute tubular necrosis 10 . Regarding intravascular hemolysis; PNH is a rare disorder and can be associated with pancytopenia and may lack a myelodysplastic disease ${ }^{3}$. It can be screened with Ham test yet confirmed with flow cytometry of patient's serum erythrocytes, neutrophils and monocytes 11. Definite diagnosis of the etiology of AKI can be established by a kidney biopsy. In our patient, we elected to be conservative since; (a) initially he had severe thrombocytopenia, (b) druginduced acute interstitial nephritis and hemolytic crisis of PAN usually respond to high-dose Corticosteroids, and (c) Ham test and flow cytometry were positive. PNH is a unique form of immune-mediated hemolytic anemia induced by inadequate suppression of activated complement leading to erythrocytes, leucocytes and platelets lysis. Complement activation is part of innate immunity. Lack of suppression is due to failure to activate complement convertase by a deficient cell-anchor protein or DAF ${ }^{12}$. The latter deficiency is: (a) an acquired one since it develops as a non-malignant expansion of mutated clones of stem cells, (b) the gene mutation is an X-linked with deficiency in glycosyl phosphatidylinositol-anchored proteins (GPI-APs) viz. CD59 on erythrocytes and CD14, 24, 157 on leucocytes 11 . Such clone expansion can develop as an isolated disorder and hence is referred to as the classic form of PNH or in associated with myelodysplastic syndrome (MDS) in type II while type III presents with subclinical PNH yet with evident BM failure 3. Clinically, the disease may present with an isolated erythrocytic or pancytopenic lytic-attacks according to the extent of the initial stem cell mutation. Moreover, its paroxysmal lytic attacks depends on activation of innate immune system during infections, autoimmune diseases, lymphoprolifertive disorders, pregnancy, vaccinations and medications (hapatens) which activates complements 12 . While other cells enhance production of convertase that deactivates the activated complements; the affected clone cells in PNH are deficient from such DAF and hence are lysed during such circumstances. Our patient had PNH without MDS i.e. classic form of disease. Moreover, he had pancytopenic presentation due to complement-activation subsequent to Ciprofloxacin therapy. Fortunately, his severe hemolytic crisis was aborted with drug-discontinuation and short-term high-dose Corticosteroids. Complications were limited to pigment-induced AKI. Most patients report dysphagia, abdominal pain, dyspnea and erectile dysfunction during hemolytic crisis. The latter is attributed to spasm of the smooth muscles due to depletion of nitric acid by hemolytic products ${ }^{13}$. Forty\% of patients with PNH are susceptible to venous thrombosis ${ }^{3}$. Limb and pelvic venous thrombosis predispose to recurrent and fatal pulmonary emboli. Moreover, thrombotic disease can involve the portal vein leading to Budd-Chiari syndrome, intestinal gangrene with thrombosis of mesenteric vein and portal hypertension with pulmonary vein disease. Specific therapy of the disease and anticoagulation should be individualized for each case. As in our patient the disease was limited to a single attack and he enjoyed long-lasting remission with precautions regarding drug and vaccination-use. Some patients may need: (a) infrequent Corticosteroid therapy to avoid its longterm side effects, (b) Danozol treatment with caution due to its virilizing effect in women, (c) Eculizumab infusions if side effects, meningococcal vaccination and cost are tolerated, and lastly (d) bone marrow transplantation if donors are available 14 . At present the latter has been proposed as the definitive therapy for the disease and Eculizumab for the acute flares. PNH usually presents in adults yet extremes of age are not spared. Children with severe disease; should have stem-cell bone marrow transplantation to avoid longterm disease management or its thromboembolic complications ${ }^{15}$. The affected females are at high risk for thromboembolic disease $(20.8 \%)$ and may need life-long anticoagulation if associated with major clone disease and during pregnancy ${ }^{16}$. In conclusion; our patient had acute drug-induced hemolytic crisis associated with minor PNH clone. With drug-vigilance; no further relapses were reported and his PNH clone remained stable for 5 years.

\section{REFERENCES:}

1- Luzzatto L. "PNH from mutations of another PIG gene". Blood 2013; 122:1099-1100.

2- Medof ME, Lublin DM, Holers VM, Ayers DJ, Getty RR, Leykam JF, Atkinson JP, Tykocinski ML. Cloning and characterization of cDNAs encoding the complete sequence of decay-accelerating factor of human complement. Proc Natl Acad Sci USA 1987; 84:2007-2011.

3- Parker C, Omine M, Richards S, et al. Diagnosis and management of paroxysmal nocturnal hemoglobinuria. Blood 2005; 106:3699-3709.

4- Hill A, DeZern AE, Kinoshita T, Brodsky RA. Paroxysmal nocturnal haemoglobinuria. Nat Rev Dis Primers. 2017 May 18. 3:17028.

5- Mehta RL, Kellum JA, Shah SV, et al. Acute Kidney Injury Network: report of an initiative to improve outcomes in acute kidney injury. Crit Care 2007; 11:R31.

6- Kidney Disease: Improving Global Outcomes (KDIGO) Acute Kidney Injury Work Group. KDIGO clinical practice guideline for acute kidney injury. Kidney Int 2012; 2(suppl):1-138.

7- Ranganath LR, Jarvis JC, Gallagher JA. "Recent advances in management of alkaptonuria (invited review; best practice article)". J Clin Pathol 2013; 66:367-73.

8- Capriotti, Theresa (2016). Pathophysiology: introductory concepts and clinical perspectives. Frizzell, Joan Parker. Philadelphia. 
9- Elise S, Michael Halefom M. The clinical significance of schistocytes: A prospective evaluation of the international councel for standerdiation in hematology schistocytes guidline. Turk J Hematol 2017; 34:59-63.

10- Dvanajscak Z, Walker PD, Cossey LN, Messias NC, Boils CL, Kuperman MB, Larsen CP. Hemolysis-associated hemoglobin cast nephropathy results from a range of clinicopathologic disorders. Kidney Int 2019; 96:1400-1407.

11- Fletcher M, Sutherland DR, Whitby L, Whitby A, Richards SJ, et al. Standardizing leucocyte PNH clone detection: An international study. Cytometry Part B 2014; 86B:311-318.

12- Nesargikar PN, Spiller B, Chavez R. The complement system: history, pathways, cascade and inhibitors. Euro J Microbiol Immunol 2012; 2:103-111.
13- Rother RP, Bell L, Hillmen P, Gladwin MT. "The clinical sequelae of intravascular hemolysis and extracellular plasma hemoglobin: a novel mechanism of human disease". JAMA 2005; 293:1653-1662.

14- Patriquin CJ, Kiss T, Caplan S, Chin-Yee I, Grewal K, et al. How we treat paroxysmal nocturnal hemoglobinuria: A consensus statement of the Canadian PNH network and review of the national registry. Euro J Hematol 2018; 102:36-52.

15- van den Heuvel-Eibrink MM, Bredius RG, te Winkel ML, et al. Childhood paroxysmal nocturnal haemoglobinuria (PNH), a report of 11 cases in the Netherlands. Br J Haematol 2005; 128:571-577.

16- Tichelli A, Socie G, Marsh J, et al. Outcome of pregnancy and disease course among women with aplastic anemia treated with immunosuppression. Ann Intern Med 2002; 137:164-172. 